\title{
MILANO ECONOMICA E LE SUE STRATEGIE
}

\author{
ANGELO MOIOLI (*)
}

SunTO. - Avanzando lungo l'età moderna e fino ben addentro la fase dell'unificazione nazionale dell'Ottocento, Milano ha mostrato di mantenersi nei ranghi più elevati del popolamento urbano in Europa. Se così è avvenuto, ciò va spiegato principalmente considerando il fatto che questa città ha continuato a rappresentare uno snodo strategico di interconnessione tra la pianura padana e l'arco alpino, grazie al suo inserimento in un contesto regionale caratterizzato più che dai confini politici in cui si scomponeva, dai fattori di coesione economica che lo tenevano insieme. Anche perché ciò avveniva pur sempre dentro a un tessuto spaziale del popolamento che faceva di Milano una sorta di "magnete" di una tra le aree più urbanizzate d'Europa. Ma a renderla tale era ormai un percorso di revisione del baricentro economico su cui gravitava il centro ambrosiano frutto di una duplicità di trattamento riservato fin dal 1781 alla parte murata rispetto al suo suburbio (Corpi Santi) e superato anche dal punto di vista daziario solo nel 1898. Era stato appunto in questo circondario esterno, il più esteso del perimetro cittadino, che si era innescato nella seconda metà dell'Ottocento un processo di differenziazione produttiva culminato nell'insediamento al suo interno della sezione più cospicua dell'industria moderna locale. Qui si era così sviluppata un'intensa dinamica immigratoria che la crisi agraria degli anni '80 non poteva avere certo interrotto. E qui si era pure venuta alimentando sotto la sua spinta, una "febbre edificatoria" senza precedenti. L'espansione del costruito aveva trovato poi modo di saldarsi con le grandi opere edilizie e di urbanizzazione realizzate entro le mura della città come nel caso dell'area dell'ex Lazzaretto o dell'ex Piazza d'Armi. Gli organismi societari e bancari che le avevano assunte e condotte a termine non erano andati esenti dalla crisi del settore insorta a Milano dopo il 1890, con effetti tuttavia assai meno dirompenti rispetto a quelli verificatisi nella capitale romana. A moderarli era stato se non l'intervento di salvataggio compiuto dal Comune di Milano a favore della Cassa per la Sovvenzione dei Costruttori, un tipo di sistema bancario ben attrezzato nell'elargizione di credito commerciale o comunque dietro garanzia reale, soprattutto se fondiaria. Era però venuto intanto il momento per la città ambrosiana di accedere anche a un nuovo circuito finanziario avente i suoi punti di forza

(*) Istituto Lombardo, Università Cattolica del Sacro Cuore di Milano, Italia. E-mail: Angelo.Moioli@unicatt.it 
nell'istituzione presso di essa di due nuove banche "miste" come Comit e Credit. Si sarebbero create così le condizioni, sotto l'egida del capitale tedesco e mettendo a frutto una politica doganale sempre più orientata in senso protezionistico, per l'insorgere di una Milano industriale di matrice ormai pienamente moderna, anche se realizzatasi secondo modalità del tutto peculiari, riconducibili al modello "per ingrandimenti successivi" tratteggiato molto tempo fa da Gino Luzzato e ripreso più di recente da Luciano Cafagna.

$* * *$

ABSTRACT. - Coming to the modern age and deep inside the national unification of the 19. century, Milan mantained the highest ranking of urban populating in Europe. This can be explained considering the fact that this town has continued to be a strategical point of intersection between the Po Valley and the Alps, thanks to its integration in a regional context characterized by the factors of economic cohesion which kept it together, more than by the political borders in which it was splitted. Also because this occured inside a populating setting that made Milan a kind of "magnet" of one among the most urbanized areas in Europe. To make it such was at that point a review course of the economic hub around which the town moved, caused by the different treatment reserved since 1781 to the part within the townwall compared to its suburb (Corpi Santi) and overtaken also from a custom point of view only in 1898 . It was exactly in this suburban area, the largest of the town limits, that in second half of the nineteenth century a process of productive differentiation occurred culminating with the settlemnent of the most important section of the modern local industry. Here developed a strong immigration that the agricultural crisis of the $80 \mathrm{~s}$ couldn't stop. And here again under its influence, an incredibly "building fever" had increased. The expansion of buildings had linked with the big building construction and urban works carried out within the townwall as the area of the ex Lazzaretto or the ex Piazza d'Armi. The companies and banks that had carried them out had been affected by the sector crisis originating in Milan after 1890, nevertheless with effect far less disruptive than in Rome. Effects limited less by the rescue packages of the Milan Municipality for the Subsidy Fund of Builder, than by a kind of banking system well prepared to the distribution of commercial credit or anyway against collateral, mostly if land guarantee. However it was high time for Milan to enter in a financial circuit with strenghts in the foundation of two new mixed banks as Comit and Credit. Therefore, under the patronage of the german capital and investing in a custom policy increasingly oriented in a protectionist step, the conditions were created to the raise of a fully modern industrialized Milan, even if achieved in a very unusual way, attributed to the model of "sequential expansions" sketched out long ago by Gino Luzzato e recalled lately by Luciano Cafagna.

Milano era giunta all'appuntamento dell'Unità nazionale venendo da un passato plurisecolare di città tra le maggiori dell'Europa. Avanzando infatti lungo l'età moderna e fino ben addentro l'Ottocento, essa aveva mostrato di mantenersi nel novero dei quindici centri urbani più popolati del continente, senza per altro che le flessioni eventual- 
mente sopravvenute si traducessero in regressi irrimediabili ${ }^{1}$. Mentre invece sarebbe successo proprio così per Venezia, seppure soltanto intorno al 1850, quando non vi sarebbe più stata la possibilità di una inversione di tendenza. È bensì vero che con il Settecento il capoluogo ambrosiano aveva dovuto cedere spazio a Roma, ma pur sempre in misura tale da non pregiudicare la possibilità di un deciso recupero di posizioni seppur rinviato a metà Ottocento, cui avrebbe fatto seguito un pareggiamento di queste entro il 1900. Ciò avrebbe tra l'altro significato anche un materiale riavvicinamento a Napoli, allora rimasta al primo posto tra le città italiane e tuttavia già non più in grado di reggere il confronto, come invece per lungo tempo era stato, prima con Parigi e poi con Londra. E sarebbe poi venuto anche il momento, mezzo secolo dopo, per prendere il sopravvento anche nei suoi confronti, alla guida di un processo di urbanizzazione in sede nazionale che riflettendo quello in atto in altri Paesi industrializzati del continente, avrebbe conferito all'aggregato metropolitano milanese una quota di popolazione da collocarsi subito dopo quelle di Londra, Parigi e Mosca².

Questa persistenza di Milano per così lungo tempo nei ranghi più elevati del popolamento urbano in Europa, non può non colpire, assodato che nel suo caso non potevano essere fatte valere certe prerogative funzionali con le quali si era tentato di spiegare l'affermarsi di concentrazioni cittadine nel pieno dell'età moderna e mentre l'antico regime economico raggiungeva la sua maturità. Se si andasse infatti ad accertare fino a che punto la città ambrosiana abbia operato come capitale di uno Stato in grado di esercitare la propria sovranità su un territorio significativamente esteso, bisognerebbe concludere che per essa ciò è stato possibile fin tanto che la formazione statuale della così detta Lombardia Viscontea ha potuto conservare l'integrità dei propri confini. Ma già tra il Quattrocento e il Cinquecento non sarebbe più stato così perché allora le provincie tra Adda e Mincio sarebbero state smembrate e aggregate alla Repubblica Veneta, proprio mentre la restante

1 L. Mocarelli, Una crescita urbana fuori delle mura. Milano e i suoi Corpi Santi tra Settecento e prima guerra mondiale, in M. Folin (a cura di), Sistole/Diastole. Episodi di trasformazione urbana nell'Italia delle città, Venezia, Istituto Veneto di Scienze, Lettere e Arti, 2006, 367.

2 Cfr. per queste elaborazioni, P. Bairoch, J. Batou, P. Chèvre, La population des villes européennes de 800 à 1850, Genève, Université de Genève, 1988, 45, 278 280, 283. 
parte di quello che ormai si chiamava lo Stato di Milano sarebbe passata sotto il dominio degli Asburgo di Spagna, diventando una sezione del tutto subordinata del relativo impero. Salvo poi dover assistere, sebbene soltanto nella prima metà del Settecento, a un'ulteriore ragguardevole scorporo a favore questa volta del Regno Sabaudo e quando già l'aggregato territoriale rimasto era passato sotto il controllo degli Asburgo di Vienna. Sembrava a quel punto definitivamente tramontata la possibilità di restituire alla città il rango di capitale. Quando invece, con l'avvento dell'età napoleonica, una simile opportunità si sarebbe ripresentata all'interno tra l'altro di una nuova area politica dalle ineguagliate dimensioni rispetto al passato. Solo che si sarebbe trattato di una vicenda destinata a chiudersi entro il primo quarto dell'Ottocento, senza più analoghi sviluppi in seguito. D'altra parte si doveva pur sempre accettare di trovarsi di fronte a una realtà urbana che oltre a vedersi privata del vantaggio di essere ancora capitale, doveva scontare una collocazione geografica che privandola di uno sbocco sul mare, le precludeva la possibilità di diventare una località portuale su cui far gravitare insieme a un intenso movimento di merci per via navale, un insediamento crescente di popolazione.

La capacità di tenuta di Milano negli assetti di vertice della demografia urbana europea di antico regime andava perciò spiegata su altri piani. A cominciare innanzitutto dal fatto di collocarsi, grazie ai canali navigabili di cui aveva iniziato a dotarsi tra il Tre e il Quattrocento, al centro di un sistema di vie d'acqua interne che intersecandosi con $\mathrm{i}$ principali bacini lacuali prospicienti le Alpi, veniva a integrarsi con $\mathrm{i}$ tracciati stradali dai quali accedere ai passi montani più agevoli per valicarle $^{3}$. Traendo vantaggio dalla riduzione dei costi e dei tempi di trasporto, esso si dimostrava in grado di far convergere sui relativi percorsi importanti flussi mercantili (sia di import-export che di transito), a cominciare da quelli da e per l'Europa Centro-Settentrionale facenti capo ai tradizionali sbocchi mediterranei della valle del Po, vale a dire di Genova da una parte e di Venezia dall'altra .

3 L. Mocarelli, Costruire la città. Edilizia e vita economica nella Milano del secondo Settecento, Bologna, Il Mulino, 2008, 50-51.

4 A. Moioli, Dinamica e struttura dell'economia lombarda tra Ottocento e Novecento, in Istituto Lombardo di Scienze e Lettere, Milano e la Lombardia nella civiltà nazionale, Milano, Istituto Lombardo di Scienze e Lettere, 1995, 185-186. 
Se poi un intreccio di vie d'acqua e di terra come questo era riuscito a fare di Milano uno snodo strategico di interconnessione tra la pianura padana e l'arco alpino, era perché si trattava pur sempre di una città che traeva la propria vitalità dal suo inserimento in un contesto regionale la cui caratterizzazione si doveva più che ai confini politici nei quali si scomponeva, ai fattori di coesione economica che lo tenevano ugualmente insieme $e^{5}$. A renderlo tale era il tessuto produttivo nel quale esso si articolava, dominato com'era da una insuperata prevalenza agricola nella quale si confrontavano due sistemi tecnico-organizzativi diversamente orientati: da un lato quello "capital intensive" proprio della pianura irrigua tra Adda e Ticino, che privilegiava un indirizzo cerealicolo-zootecnico fortemente mercantilizzato; dall'altro quello "labour intensive" nel quale alla piccola coltura contadina a grani si accompagnavano opzioni a valenza commerciale dipendenti dallo sfruttamento del soprasuolo nei seminativi, la principale delle quali era data sempre più dalla gelsibachicoltura ${ }^{6}$. Ed era ancora attraverso l'agricoltura che traeva il proprio sviluppo una porzione cospicua dell'attività manifatturiera esercitata in quello stesso complesso territoriale, seppure in modo variamente polarizzato tra città e campagna. Se non altro perché da essa derivavano in gran parte le materie prime da trasformare in semilavorati e prodotti finiti, quando non anche la mano d'opera rurale da impiegare in alcune fasi della loro lavorazione a domicilio.

Una compagine agricolo-manifatturiera come questa che alimentava correnti di interscambio sia all'interno delle coordinate spaziali entro cui si sviluppava, sia al loro esterno in sede internazionale, presupponeva l'articolarsi di una domanda di beni di consumo e di produzione resa interattiva secondo un duplice ordine di circuiti di mercato: l'uno riconducibile a una dimensione infraregionale della sua dinamica; l'altro invece incentrato sui rapporti commerciali con l'estero. In entrambe le direzioni era però il grado di urbanizzazione del territorio di riferimento ad assumere un ruolo decisivo. Laddove infatti prendevano il sopravvento insediamenti di popolazione concentrati in città, si

5 Id., Assetti manifatturieri nella Lombardia politicamente divisa della seconda metà del Settecento, in S. Zaninelli (a cura di), Un sistema manifatturiero aperto al mercato, Milano, Il Polifilo, 1988, 4-5.

6 Id., La Lombardia: una dimensione regionale dello sviluppo, in Carlo Cattaneo. Scritti sulla Lombardia, Milano, Oscar Mondadori, 2002, XL-XLI. 
davano anche le condizioni per l'affermarsi prioritario al loro interno di una economia votata allo scambio su basi monetarie, quale si sarebbe ben poco riscontrata in presenza di un popolamento a eminente impronta rurale. E a maggior ragione ciò era sostenibile proprio nel caso della Lombardia, dove già nel corso dell'età di mezzo si era venuta configurando una delle aree più urbanizzate d'Europa. Milano ne era, come è stato ben detto, "il magnete" era consolidato un sistema policentrico di altre città tra loro interrelate, le cui dimensioni potevano anche raggiungere entità considerevoli.

Nè è da credere che la maggiore città lombarda fosse riuscita a mantenere una più elevata taglia di popolazione, per averla comunque accresciuta a scapito degli altri centri urbani con i quali interagiva entro la medesima area regionale. Ma se così fosse stato, ciò avrebbe voluto dire il celarsi dietro la primazia milanese di una egemonia in grado di smentire l'esistenza di un equilibrio di posizioni tra detta città e il restante complesso di quelle circostanti delle più popolate 8 . Quando invece le stesse andavano mostrando come le loro capacità di tenuta nei primi posti del popolamento regionale si accompagnasse al mantenimento di una vitalità economica tutta speciale, in grado di autoalimentarsi in piena autonomia almeno per tutto il corso dell'età moderna, se non anche più tardi.

La forza di attrazione della città ambrosiana si veniva dunque esprimendo senza che la preminenza di questa si risolvesse in una polarizzazione del proprio ruolo sino a sminuire quello più proprio del reticolo urbano che la contornava sul piano regionale. Non ne risentiva tuttavia la sua conformazione che sarebbe rimasta così despecializzata almeno sino a quando l'avvento dell'industria moderna si fosse affermata pienamente al suo interno. Perché soltanto allora si sarebbero innescati processi specializzanti a filiere integrate dal lato manifatturiero e dei servizi in grado di generare una "Milano industriale" diversa, se non ancora alternativa, rispetto a quella che Giuseppe Colombo avrebbe rappresentato in un saggio giustamente famoso del 1881, assumendo la parigina "città atelier" come modello di un insediamento produttivo a impianto disseminato della manifattura'. Vi si accompagnava comunque un assetto del popola-

7 L. Mocarelli, Costruire la città, cit., 60.

8 Ivi, 47.

9 E. Borruso, Dal laboratorio artigiano alla piccola impresa urbana. Appunti 
mento di tipo radiocentrico, distribuito su tre fasce di territorio ad estensione crescente, la prima delle quali quella centrale, racchiusa entro la cerchia dei navigli, denotava la più alta densità insediativa proprio per raccogliervi, insieme alla maggior quota di abitanti, anche la parte più cospicua del tessuto economico cittadino a base non agricola ${ }^{10}$. Ma ormai si stavano creando le condizioni per dilatare la superficie urbanizzata oltre la conca interna fino all'aggregato intermedio delimitato dalle mura spagnole, e sfociando poi nella grande sezione periferica dei Corpi Santi. E sarebbe stata proprio questa l'area dove, a partire dai decenni centrali dell'Ottocento, si sarebbe avviata una revisione del preesistente baricentro economico su cui gravitava Milano fin da tempi lontani ${ }^{11}$. A renderla tale avrebbe allora concorso "la duplicità" di trattamento cui questa era stata sottoposta già nel 1781 dal punto di vista politico-amministrativo e soprattutto fiscale, per via dell'attribuzione ai Corpi Santi della qualifica di comune separato da quello della città murata. Ė bensì vero che questa prerogativa sarebbe venuta meno nel 1873, quando la "consorteria" liberaldemocratica alla guida di Palazzo Marino era riuscita a ottenere con decreto reale la fusione tra i due organismi comunali. Ma è un fatto che solo a un decennio di distanza si sarebbe riusciti a porre in essere una effettiva unificazione elettorale tra loro $^{12}$. Inoltre proprio mentre la città "esterna" si vedeva costretta, seppure dopo una lunga contesa, a cedere le armi del potere decisionale a quella "interna", era riuscita comunque a protrarre fino al 1898 l'esistenza a proprio vantaggio di un duplice regime daziario, che il sindaco Gaetano Negri nel 1886, non senza suscitare gravi tensioni sociali, aveva cercato di modificare a vantaggio della città murata, introducendo il cosiddetto "dazio della micca", a carico degli abitanti del suburbio costretti a pagarlo andando a lavorare nel circondario interno ${ }^{13}$.

sull'esperienza milanese (1881-1921), C. M. Belfanti, F. Maccabelli (a cura di), Un paradigma per i distretti industriali. Radici storiche, attualità e sfide future, Brescia, Grafo, 1997, 80.

10 L. Mocarelli, Costruire la città, cit., 34.

11 Id., Una crescita urbana fuori delle mura, cit., 373.

12 G. Vecchio, La classe politica milanese nello Stato liberale. I moderati (18701900), in C. Mozzarelli, R. Pavoni (a cura di), Milano fin de siècle e il caso Bagatti Valsecchi. Memoria e progetto per la metropoli italiana, Milano, Guerini e associati, 1991, 273.

13 M. Punzo, L'amministrazione della città, in G. Rumi, A. C. Buratti, A. Cova (a cura di), Milano nell'Unità nazionale 1860-1898, Milano, Cassa di Risparmio delle Province Lombarde, 1991, 420-421. 
Ma c'è di più perché mentre veniva meno il trattamento di favore riservato al circondario della città non murata, questo trovava modo di assumere una rilevanza economica ben maggiore di quella che in precedenza era ad esso derivata da una prioritaria destinazione della relativa dotazione fondiaria all'esercizio di una agricoltura mercantile votata ai consumi alimentari di quello stesso contesto urbano e riflettente l'assetto tecnico-organizzativo della pianura irrigua lombarda in cui si integrava. Era in atto infatti al suo interno un processo di differenziazione produttiva indotto dall'insediamento in loco di una crescente parte del nuovo tessuto industriale emergente a Milano nella seconda metà dell'Ottocento. Qui si davano per altro vantaggi localizzativi di grande impatto, quali l'abbondanza di suolo edificabile a buon mercato, l'accesso più libero e meno costoso a fonti di energia idraulica senza le restrizioni imposte invece entro le mura spagnole ${ }^{14}$, in aggiunta alla crescente disponibilità di mano d'opera di estrazione rurale che vi andava affluendo dalle località circostanti. Dal loro combinarsi emergeva una forza di attrazione di quel circondario condensatasi in una crescita della popolazione locale dai ritmi incalzanti, che l'avrebbero vista moltiplicarsi di ben ventisei volte tra il 1816 e il 1911, sino a raggiungere e superare sul finire dell'Ottocento la consistenza assegnabile all'intera città murata ${ }^{15}$. Vi si sarebbe così addensato un numero di abitanti certamente da primato che ai primi del Novecento aveva raggiunto la bella cifra di 622 individui per ha, quando invece entro la cerchia dei navigli non si era andati oltre i $563^{16}$. Ciò dipendeva anche dal fatto che trattandosi di realizzare nuovi grandi complessi industriali, questi si preferiva ormai collocarli fuori dai bastioni e non tanto perché nella città che vi era inclusa mancassero gli spazi necessari, quanto invece a motivo del rendersi sempre più costosi quelli disponibili.

A imporlo erano le stesse ristrutturazioni del tessuto urbanistico che si andavano compiendo. Queste non si erano certo limitate ai rifacimenti di Piazza del Duomo e della Scala, unitamente all'erezione della Galleria intestata a Vittorio Emanuele $\mathrm{II}^{17}$. Portata infatti a termine con

14 L. Mocarelli, Una crescita urbana fuori delle mura, cit., 385.

15 Ivi, 372.

16 Ivi, 373.

17 V. Vercelloni, La trasformazione urbana, in G. Rumi, A. C. Buratti, A. Cova (a cura di), Milano nell'Unità nazionale, cit., 153. 
queste opere la sistemazione del centro città, altre se ne erano poi aggiunte volte a dilatare i contorni dello stesso, affidandosi per questo alla realizzazione di nuovi quartieri residenziali in una con la riqualificazione di quelli preesistenti, ad uso di abitazioni borghesi e/o di luoghi del terziario. Di conseguenza, in questa parte dello spazio urbano, i valori delle aree edificabili avevano iniziato a lievitare, trascinando verso l'alto anche i prezzi delle locazioni. Aveva allora guadagnato in convenienza la scelta di uscire dai bastioni per andare a collocare nella cerchia ad essi esterna sia fabbricati industriali, sia edifici volti ad ospitare abitazioni in affitto. Ed era stato così che aveva preso sempre maggior risalto l'area edificata dei Corpi Santi, mettendo per questo a frutto anche i terreni altrimenti riservati a una destinazione agricola. Come è stato fatto notare, già nel decennio 1871-1881 si calcolava che vi fossero sorti ben 500 edifici adibiti ad alloggio, quando invece nello stesso periodo ed entro le mura spagnole se ne erano contate appena 64 dello stesso tipo ${ }^{18}$. Vi si andava dunque manifestando, seppure ancora in una fase iniziale, quella che già allora veniva definita una "febbre edificatoria".

Nè la crisi agraria degli anni ' 80 - anche ammesso che essa avesse avuto per l'economia milanese gli stessi effetti depressivi cui ha fatto largamente riferimento la storiografia nazionale ${ }^{19}$ - sembra essere stata tale da far inceppare l'espansione edilizia in corso nel suburbio. Se non altro perché essa avrebbe contribuito a far dirottare ancor più l'eccesso di mano d'opera che si andava accumulando nelle campagne, verso le periferie della città ambrosiana. $\mathrm{E}$ perciò in quel contesto la domanda abitativa e di fabbricati aventi altra destinazione non conosceva arresti significativi e l'offerta edilizia continuava quindi a prosperare. Tant'è che già nel 1873, all'indomani del ricongiungimento in una unica municipalità della città murata con quella del circondario esterno ${ }^{20}$, era stato proprio il dilatarsi delle superfici edificate all'interno di quest'ultimo a far ritenere che fosse giunto il momento di realizzarvi un piano regolatore in grado di assicurare allo stesso uno sviluppo degli insediamenti

18 L. Mocarelli, Una crescita urbana fuori delle mura, cit., 380.

19 Cfr. M.L. Cavalcanti, L'economia italiana in età liberale: una rivoluzione storiografica?, in F. Balletta (a cura di), Il pensiero e l'opera di Domenico Demarco, Milano, F. Angeli, 2010, 204-209, con A. Baffigi, Il PIL per la storia d'Italia. Istruzioni per l'uso, Venezia, Marsilio, 2015, 28-29.

20 L. Mocarelli, Una crescita urbana fuori delle mura, cit., 378. 
più ordinato e meno esposto ad abusi ed eccessi. Sarebbero passati comunque tre anni prima di portare a termine un tale progetto ${ }^{21}$, ma quando ciò era avvenuto aveva pur sempre voluto dire per il circondario esterno mettersi in una posizione di avanguardia rispetto al resto della città, che sarebbe rimasta priva ancora per diversi anni di una analoga strumentazione urbanistica. Dentro i bastioni si era però trovato ugualmente modo nel frattempo di realizzare piani di edificazione parziali, secondo una procedura che ne addossasse la formulazione ai privati titolari delle stesse, da presentare agli uffici municipali competenti. Vi faceva seguito la stipula tra le parti di speciali convenzioni volte a definire le opere di urbanizzazione a carico dei costruttori e i criteri di ripartizione dei relativi oneri, affinché si giungesse a una loro approvazione definitiva in sede consigliare ${ }^{22}$. E così in effetti era stato quando la Banca di Credito Italiano dopo il suo trasferimento a Milano da Torino nel 1875 e tramite la Compagnia Fondiaria Italiana che ne era l'emanazione, provvedeva con il 1881 ad acquistare dagli Enti Ospedalieri Milanesi l'edificio e l'area del Lazzaretto di Porta Venezia da destinare alla realizzazione di un nuovo quartiere ${ }^{23}$. Di questo, che si sarebbe caratterizzato per l'alta densità del costruito $^{24}$, era stata poi presentata alla Giunta Municipale una proposta di piano regolatore, oggetto di una convenzione approvata dal Comune il 21 gennaio 1883. Sempre in quello stesso anno sarebbe stata anche la volta della lottizzazione, affrontata pur essa in regime di convenzione, relativamente ai terreni di proprietà dei banchieri svizzeri Waill-Schott, nella zona compresa tra via Monforte e Porta Romana ${ }^{25}$.

21 M. Tiepolo, Promotori e operazioni immobiliari a Milano durante il piano Beruto (1884-1894), in R. Rozzi (a cura di), La Milano del piano Beruto (1884-1889). Società urbanistica e architettura nella seconda metà dell'Ottocento, Milano, Guerini e Associati, 1992, 114.

22 A. Polsi, Banche e banchieri a Milano nella seconda metà dell'Ottocento, in C. Mozzarelli, R. Pavoni (a cura di), Milano fin de siècle e il caso Bagatti Valsecchi, cit., 340-341.

23 M. Tiepolo, La formazione del quartiere dell'ex Lazzaretto a Milano (18821892): un'operazione della Banca di Credito Italiano, in "Storia della Lombardia", Milano, a. VII (1988), n. 1, 27-30.

24 A. Polsi, Élite finanziaria e comune a Milano nella seconda metà dell'Ottocento. Ipotesi per una ricerca, in S. Adorno, C. Sorba, Municipalità e borghesia padana tra Ottocento e Novecento, Milano, F. Angeli, 1991, 30.

25 M. Tiepolo, Promotori e operazioni immobiliari a Milano, cit., 121. 
Vi avrebbe poi fatto seguito una operazione immobiliare di ancor più ampio rilievo, consistente nella urbanizzazione della vecchia Piazza d'Armi, ora che questa era entrata a far parte del demanio comunale. Giunta nel vivo della sua attuazione a partire dal 1884, aveva suscitato bensì, in merito ai piani dettagliati di edificazione e agli accordi in materia di viabilità che vi si erano accompagnati, forti opposizioni all'interno del consiglio comunale. Esse tuttavia, se anche sfociate entro l'aprile di quell'anno, nelle dimissioni del sindaco Giulio Belinzaghi e della sua Giunta $^{26}$, non avevano certo inceppato più di tanto il procedere dei grandi lavori intrapresi. Era stato allora importante già il contributo venuto dalla Società Fondiaria Milanese costituitasi nel 1881 a Milano avendo tra i suoi maggiori soci fondatori i francesi dell'Union Générale, unitamente alla Banca Generale e alla Banca di Milano. Fallito poi l'anno dopo l'istituto francese e fusasi allora la Banca di Milano che esso prima controllava con la Subalpina di Torino, quest'ultima aveva finito per lasciare alla Banca Generale il controllo della Fondiaria ${ }^{27}$. Questa stessa banca vi avrebbe poi affiancato la Cassa di Sovvenzione dei Costruttori a partire dal 1886, fondata bensì a Roma, ma subito trasferita con la propria sede a Milano, per assumervi un rilievo strategico di intervento speculativo nella zona della nuova via Dante e del Foro Bonaparte ${ }^{28}$.

Alcuni di questi organismi societari e bancari si stavano per altro esponendo, seppure in varia misura, nell'acquisizione di vaste aree edificabili situate nella cerchia suburbana della città, ma non più riconducibili al piano regolatore abbozzato nel 1876 per quel territorio, come si è detto. Sul finire del 1883 si era deciso infatti di ricomprenderlo in una formulazione più complessiva, riferita all'intero comprensorio cittadino, dentro e fuori le mura. Si trattava del ben noto piano Beruto, dal nome del capo dell'ufficio tecnico comunale che l'aveva impostato dietro incarico del neo-sindaco Gaetano Negri, dopo l'elezione di questi nel gennaio 1885, ma poi ugualmente sottoposto a non poche modifiche da una commissione municipale presieduta da Giovanni Battista Pirelli²

26 M. Punzo, L'amministrazione della città, cit., 420.

27 M. Tiepolo, Promotori e operazioni immobiliari a Milano, cit., 120.

28 A. Dotti, Gli istituti di credito in Milano. Gli istituti di credito nel primo trentennio unitario, in Storia di Milano, vol. XV, 1859-1900, Milano, Fondazione Treccani degli Alfieri, 1962, 999-1001.

29 M. Punzo, L'amministrazione della città, cit., 420. 
La sua versione definitiva sarebbe poi stata fatta valere tenendo distinta la sezione murata da quella del suburbio. Non per niente la rilevazione di quest'ultima era stata presentata come un "ampliamento" della prima, essendo stata oggetto tra l'altro di una approvazione ministeriale più tarda di quella riservata al resto di tale contesto urbano ${ }^{30}$. L'aver comunque ricondotto a un unico disegno pianificatore i due assetti fondiari costitutivi dello stesso, aveva però intanto consentito di verificare se e in che misura la grande speculazione immobiliare si stesse proiettando anche verso la fascia suburbana. Stando a una indagine compiuta di recente proprio a questo specifico riguardo, gli 11 soggetti detentori dei maggiori patrimoni fondiari accumulati in zona tra il 1884 e il 1889 giungevano a totalizzare intorno a $640 \mathrm{ha}^{31}$, che corrispondeva pur sempre al $10 \%$ circa della superficie complessiva di quel territorio (pari a $6225 \mathrm{ha})^{32}$. Non era una gran cifra e tuttavia quel che conta è il rilevare come a dimensionarla in misura nettamente prevalente era innanzi tutto la quota di terreni assegnata alla Società Fondiaria Milanese, cui si aggiungeva, seppure a distanza, quella spettante alla Cassa di Sovvenzione dei Costruttori che l'aveva gemmata. Si delineava perciò un intreccio di scelte che mentre affidava a detta società la funzione centrale di investitore immobiliare proiettata oltre i bastioni, ne faceva assumere una analoga, seppure in via subordinata, alla stessa Cassa, benché quest'ultima restasse principalmente votata alla valorizzazione urbanistica della ex Piazza d'Armi. Un analogo mix di combinazioni si andava intanto ripetendo, seppure in proporzioni più contenute, tra acquisizioni di nuovi spazi compiute nei Corpi Santi da parte della Banca di Credito Italiano e in stretta connessione con la Compagnia Fondiaria Italiana, proprio mentre entrambe si andavano sempre più esponendo nella grande lottizzazione dell'ex Lazzaretto ${ }^{33}$.

Il congiunto posizionarsi di questi investitori nel controllo delle aree edificabili dentro e fuori la cerchia muraria sarebbe diventato poi un ulteriore motivo di differenziazione nei ritmi di intensificazione del costruito e a beneficiarne ancor più era di nuovo il circondario esterno. Se ne aveva la riprova mettendo a confronto per il 1890 la dotazione di

30 M. Tiepolo, Promotori e operazioni immobiliari a Milano, cit., 124.

31 Ivi, 121.

32 L. Mocarelli, Una crescita urbana fuori delle mura, cit., 371.

33 M. Tiepolo, Promotori e operazioni immobiliari a Milano, cit., 120. 
locali realizzati in quell'anno al di qua e al di là dei bastioni: 4137 vani sul primo fronte contro 6758 sul secondo. E addirittura l'anno dopo, dinnanzi ai cali allora registratisi, nell'ordine rispettivamente del $56 \%$ e del $38 \%$, non si era certo assistito a un deciso ribaltamento di posizio$\mathrm{ni}^{34}$. Era quello soltanto il segnale della crisi edilizia in corso anche a Milano dove per altro i suoi effetti sarebbero apparsi meno eclatanti che non a Roma. Può anche darsi, come in effetti è stato sostenuto, che la maturazione dello slancio impresso al settore sia stata più rapida che non nella capitale, in presenza di una struttura fondiaria del suburbio ancora frazionata a tal punto da impedire il formarsi di situazioni patrimoniali egemoni in grado di alimentare spinte speculative rovinose ${ }^{35}$. Ma è un fatto comunque che quando nel 1892 la Banca di Credito Italiano più sopra nominata era stata messa in liquidazione, ciò aveva riguardato pur sempre un istituto che impegnato nella realizzazione di un progetto edilizio di speciale rilievo per l'Ottocento milanese, qual era stato quello del Lazzaretto ${ }^{36}$, aveva mostrato di saperlo fare con successo, se è vero che sino ad allora aveva portato a compimento la vendita di ben 1'88\% degli stabili in questione ${ }^{37}$. Tanto più che si era trattato di un esito ottenuto anche per effetto delle agevolazioni accordate ai costruttori in ordine ai crediti loro concessi sia per l'acquisto dei terreni, sia per lo svolgimento dei lavori di edificazione ${ }^{38}$. D'altra parte è ugualmente significativo che la Compagnia Fondiaria Italiana, detentrice insieme a tale banca di un grosso patrimonio fondiario nelle zone suburbane, sia giunta a smaltirlo ancor prima di giungere al suo scioglimento $^{39}$. Nè è da credere che proprio a Milano avrebbe potuto assumere una forte valenza destabilizzante la chiusura degli sportelli toccata a una grande società di credito mobiliare come la Banca Generale, avvenuta nel 1894, a un anno di distanza da quella della ancor più importante Società Generale di Credito Mobiliare. È bensì vero che tale istituto, sorto per iniziativa principalmente di banchieri milanesi, aveva poi sviluppato la propria molteplice attività speculativa oltre che a Roma e a Napoli, sulla stessa piazza ambrosiana, privilegiando anche in quella

\footnotetext{
34 Ivi, 125.

35 Ivi, 121.

36 A. Polsi, Élite finanziaria e comune a Milano, cit., 29.

37 M. Tiepolo, La formazione del quartiere dell'ex Lazzaretto a Milano, cit., 21.

38 A. Polsi, Élite finanziaria e comune a Milano, cit., 30.

39 M. Tiepolo, Promotori e operazioni immobiliari a Milano, cit., 126.
} 
sede gli impieghi in campo edilizio ${ }^{40}$. Ma vi si era pur sempre esposta dando il suo apporto determinante alla costituzione in loco di quella Cassa per la Sovvenzione dei Costruttori che non avrebbe certo potuto evitare di cadere nella spirale della crisi del settore e tuttavia riuscendo ugualmente a pararne le conseguenze più pesanti. Spettava infatti a detta società il compito di condurre in porto la vasta operazione di lottizzazione e urbanizzazione in corso a Piazza d'Armi e dintorni e la convenzione da essa stipulata con il comune nel 1887, all'indomani del suo trasferimento a Milano da Roma, sembrava la più adeguata ai fini del pieno successo di tale iniziativa ${ }^{41}$. Quando invece già nel giro di un triennio, le crescenti difficoltà di collocamento degli stabili messi in vendita, con conseguente appesantimento della situazione debitoria, avevano fatto rilevare ben presto pesanti perdite di bilancio ${ }^{42}$. Si stava andando verso uno stato di insolvenza e questo sarebbe divenuto inevitabile se non si fosse riusciti nel frattempo almeno a sanare le sofferenze insorte con il comune di Milano, suo principale creditore. Ed ecco allora emergere la proposta che fosse proprio quest'ultimo a doversi "ricomprare parte dei terreni che in passato aveva ceduto alla Cassa e che non gli erano stati pagati". Si trattava dei 93 ha tra Foro Bonaparte e la Piazza d'Armi che la società aveva acquistato due anni prima per la bella cifra di 4 milioni di lire ${ }^{43}$. Era stata stilata per questo anche una convenzione e la Cassa si era così liberata dal gravoso debito assunto da tempo verso l'ente suo creditore. Anche se poi ciò non era bastato ad evitare che essa venisse messa in liquidazione dal 1892. Ma a quel punto ormai la portata della sua crisi che pur perdurava, non era più tale da amplificarne gli effetti sull'intero comparto edilizio della città.

Questa sorta di salvataggio ante litteram allora affrontato non deve far credere che il Comune milanese stesse per abbandonare la posizione di sostanziale neutralità in precedenza tenuta verso l'iniziativa privata operante nel settore delle costruzioni ${ }^{44}$. Al massimo si trattava di assecondare lo sviluppo di questa e non certo per sostituirsi ad essa. In effetti

40 A. Dotti, Gli istituti di credito in Milano, cit., 999.

41 M. Tiepolo, Promotori e operazioni immobiliari a Milano, cit., 126.

42 A. Dotti, Gli istituti di credito in Milano, cit., 1001.

43 M. Tiepolo, Promotori e operazioni immobiliari a Milano, cit., 126.

44 A. Polsi, Banche e banchieri a Milano nella seconda metà dell'Ottocento, cit., 340-341. 
se la crisi edilizia aveva potuto essere qui contrastata più efficacemente che altrove non era tanto per il fatto che il Comune ambrosiano era diventato più interventista, almeno dopo che si era dotato di un piano regolatore come quello Beruto, quanto invece perché, in presenza di un ciclo di recupero dei capitali investiti nelle opere di edificazione in corso, di per sé reso più prolungato per i tempi di lavorazione praticati ${ }^{45}$, il sistema bancario vigente appariva pur sempre il più attrezzato nell'assicurare l'elargizione di credito a condizioni facilmente accessibili, mettendo a frutto per questo l'elevato grado di liquidità di cui era dotato ${ }^{46}$. Di modo che la sua forza crescente dal lato della raccolta di risparmio venisse finalizzata il più possibile alla concessione di prestiti agli stessi costruttori, affidata alla emissione di cambiali a due o tre firme con la scadenza al massimo di sei mesi, comunque rinnovabili attraverso il risconto degli effetti presso la Banca Nazionale e relative succursali ${ }^{47}$. Suoi punti di forza erano da un lato i banchieri privati che proprio in questa città restavano specialmente numerosi, ma anche e soprattutto intenti a "diversificare i rischi su vari piani, complementari e non concorrenziali tra loro" ${ }^{48}$. Vi si affiancavano da un lato la Banca Popolare di Milano e dall'altro la Banca Lombarda di depositi e conti correnti che proprio negli anni '80 e '90 si venivano distinguendo per la loro partecipazione alle operazioni di sconto seppure con margini variamente rilevanti $^{49}$. Nè era da meno la stessa Cassa di Risparmio che, come è stato fatto notare, "attraverso il proprio credito fondiario, non poteva non essere coinvolta nella febbre edilizia" da cui era stata investita la città. Parrebbe infatti che dalla seconda metà degli anni '80 l'erogazione di mutui fondiari fosse in notevole aumento, con un raddoppio raggiunto nel 1887 e con un elevato livello di prestiti ipotecari mantenutosi tale fino ai primi anni '90. Tra l'altro ciò era avvenuto sempre di più dietro garanzia di beni "urbani" e a decrescere di quelli "rurali"

Una struttura creditizia così combinata si guadagnava per altro il

45 A. Dotti, Gli istituti di credito in Milano, cit., 995.

46 Ivi, 1002.

47 A. Polsi, Banche e banchieri a Milano, cit., 338.

48 Ivi, 337.

49 A. Dotti, Gli istituti di credito in Milano, cit., 996.

50 P. Cafaro, Finanziamento e ruolo della banca, in S. Zaninelli (a cura di), Storia dell'industria lombarda. Alla guida della prima industrializzazione italiana. Dall'unità politica alla fine dell'Ottocento, Milano, Il Polifilo, 1990, 240-241. 
suo rilievo non solo sul fronte edilizio, ma anche su quelli che stavano qualificando ben altrimenti la centralità del tessuto economico milanese ormai sul piano nazionale. Ma lo poteva fare in quanto, una volta esaurite le esperienze bancarie finalizzate alla speculazione sui suoli urbani di cui si è detto, stava venendo per essa il momento di accedere a un nuovo circuito finanziario diversamente orientato all'esercizio della liquidità $\mathrm{e}$ in vista di impieghi volti ad alimentare le iniziative del comparto industriale nella sua valenza più moderna. Ed era la volta adesso di un deciso intervento del capitale tedesco ${ }^{51}$, affluito quest'ultimo attraverso investimenti sia di portafoglio che diretti $i^{52}$. Suo punto di forza era pur sempre quello di avvenire come emanazione del sistema di "banche miste" su cui poggiava già allora il successo economico della Germania e trovando modo di manifestarsi in quella versione anche a Milano, dove rispettivamente nel 1894 e nel 1895 erano sorti due istituti come Comit e Credit in grado da subito di riprodurre efficacemente la formula, se non altro dal punto di vista tecnico-manageriale ${ }^{53}$. Era stato tramite loro infatti che la piazza finanziaria ambrosiana era uscita dalla posizione subordinata in cui prima si trovava, in vista di una supremazia sul piano nazionale che in seguito non sarebbe più venuta meno. E potrebbe anche essere che a un simile esito si fosse giunti per effetto di "una sorta di campagna d'Italia" ${ }^{54}$ condottavi dal capitale germanico nel mutato clima politico indotto dall'adesione italiana alla Triplice Alleanza. Ma è un fatto che ciò capitava proprio mentre con l'area tedesca era in atto un interscambio destinato in prospettiva a dilatarsi, facendo di Milano "il maggior tramite commerciale e di affari tra Italia e Germania"55.

51 Cfr. P. Hertner, Il capitale tedesco in Italia dall'Unità alla Prima Guerra Mondiale, Bologna, Il Mulino, 1983.

52 Per i necessari distinguo tra queste tipologie di investimento, cfr. Id., Introduzione, a Investimenti, tecnologie e capitale umano di origine straniera, in «Padania», Ferrara, a. II (1988), 9-10, con G. Roggero-Fossati, I movimenti internazionali di capitale, Milano, Giuffrè, 1972, 9.

53 A. Confalonieri, Banca e industria in Italia (1894-1906), Bologna, Il Mulino, 1980, 9-50. Ma cfr. anche A. Cova, Milano crocevia della finanza, in G. Rumi, A. C. Buratti, A. Cova, Milano nell'Italia liberale, cit., 323.

54 P. Pecorari, L'Italia economica. Tempi e fenomeni del cambiamento. $1861-$ 2000, Padova, CEDAM, 2005, 53.

55 A. Sapori, L'economia milanese dal 1860 al 1915, in Storia di Milano, vol. XV, cit., 871 . 
A maggior ragione ciò si doveva sostenere ora che, a partire dal 1882, era stata attivata la ferrovia internazionale del Gottardo. Se ne era tanto convinti che quando si era trattato di conferire il capitale iniziale dell'impresa, era stato proprio a Milano che si era raccolta la metà degli stanziamenti posti a carico dell'Italia, pari nel complesso a 55 milioni di lire $^{56}$. Del resto i volumi di traffico convogliati su questa via di accesso ai mercati centro-europei sarebbero stati tali da assegnare al polo ferroviario di questa città un rilievo sul piano nazionale in precedenza mai raggiunt ${ }^{57}$. Era in gioco però ormai anche una revisione strategica della regolazione doganale da parte italiana nei confronti dei principali partners commerciali dell'Europa Continentale e quindi della stessa Germania. A cominciare dalla tariffa generale riformata dal 1878. Essa, introducendo dazi specifici rispetto a quelli preesistenti ad valorem, veniva instaurando un regime moderatamente protezionista ${ }^{58}$, a fronte del quale proprio a Milano si venivano contrapponendo ora ai liberisti i protezionisti, se non altro come fautori del "germanesimo economico" allora in auge ${ }^{59}$. Sede principale di questo confronto era ormai diventata la locale Camera di Commercio, anche se per il momento ciò stava avvenendo senza contrapposizioni particolarmente accese. Posto del resto che i vantaggi recati dalla revisione tariffaria in corso si estendessero a tutta la Penisola, è un fatto che essi trovassero modo di manifestarsi più pienamente proprio a Milano e nella regione circostante, il cui contesto economico presentava attributi di solidità e di differenziazione settoriale tali da accentuarne i momenti di prosperità e da moderarne quelli di crisi ${ }^{60}$. Ma quando all'indomani dell'abolizione del corso forzoso nel 1883, si era imposta la necessità di rinnovare i trattati commerciali in scadenza sul piano europeo, a cominciare da quello con la

56 E. Dalmasso, Milano capitale economica d'Italia, Milano, F. Angeli, 1970, 194-195.

57 G. Luzzatto, L'evoluzione economica della Lombardia dal 1860 al 1922, in La Cassa di Risparmio delle Provincie Lombarde nell'evoluzione economica della regione, 1823-1923, Milano, Alfierie Lacroix, s.d. (1923), 482.

58 P. Pecorari, Il protezionismo imperfetto. Luigi Luzzatti e la tariffa doganale del 1878, Venezia, Istituto Veneto di Scienze, Lettere e Arti, 1989, 370-391.

59 A. M. Galli, Credito e finanza. La Cassa di Risparmio e la crescita economica e sociale, in G. Rumi, A. C. Buratti, A. Cova (a cura di), Milano nell'unità nazionale, cit., 446.

60 E. Dalmasso, Milano capitale economica d'Italia, cit., 182. 
Francia $^{61}$, quello stesso organo camerale si era dimostrato incapace di una mediazione degli interessi in esso rappresentati simile a quella affrontata in occasione delle modifiche tariffarie del 1878.

In effetti era prevalso al suo interno un incondizionato sostegno alla scelta protezionistica che il governo italiano aveva fatta propria e che lo avrebbe condotto nel 1887 alla rottura dei rapporti commerciali con l'antagonista francese ${ }^{62}$. Avendo però ciò significato allora una contrapposizione tra le ragioni degli industrialisti volti al controllo in chiave protezionistica del mercato interno e gli interessi libero-scambisti più consolidati quali erano quelli degli esportatori serici, non era stato possibile pervenire a una conciliazione delle rispettive posizioni. Si era anzi giunti a una frattura tale da provocare il commissariamento dall'ente camerale già nel corso del $1888^{63}$. Esso avrebbe se non altro favorito il manifestarsi di valutazioni più ponderate circa l'opportunità del mantenimento di una tutela doganale come quella in corso, ma niente di più. E intanto erano venute meno le alleanze che in precedenza, per almeno un trentennio, avevano retto le sorti della vicenda camerale a Milano. Si era assistito così anche alla scissione verificatosi all'interno del Circolo Industriale Commerciale e Agricolo che tanta parte aveva avuto come punto di riferimento unitario per la composita borghesia cittadina $^{64}$. Ad abbandonarlo erano ora in special modo gli esponenti più qualificati del ramo serico, confluiti nel nuovo Circolo per gli interessi industriali, commerciali ed agricoli con l'obiettivo di contrastare l'indirizzo "ultraprotezionista" imposto con l'adozione della tariffa autonoma dal 1887, che li costringeva a cercarsi altri mercati di sbocco rispetto a quello francese. Sarebbero stati loro a dar vita poi a quella "Associazione per la libertà economica" cui avrebbero aderito in pro-

61 S. Zaninelli, Aspetti economico-produttivi, di mercato e tecnologici, in Id. (a cura di), Storia dell'industria lombarda. Alla guida della prima industrializzazione italiana, cit., 113.

62 E. Decleva, Milano industriale e l'Esposizione Nazionale di Milano, in Id. (a cura di), L'Italia industriale nel 1881. Conferenza nella Esposizione Nazionale di Milano, Milano, Banca del Monte, 1984, XXXI.

63 N. Crepax, La Camera di Commercio di Milano durante gli ultimi decenni dell'Ottocento, in C. Mozzarelli, R. Pavoni (a cura di), Milano fin de siècle, cit., 271.

64 A. Moioli, La cultura economica della borghesia produttiva milanese attraverso i periodici specializzati (1890-1914), in P. L. Porta (a cura di), Milano e la cultura economica nel XIX secolo. Gli anni 1890-1920, Milano, F. Angeli, 1998,25. 
sieguo di tempo autorevoli economisti come Ugo Mazzola, Vilfredo Pareto, Ulisse Gobbi, Antonio De Viti De Marco ${ }^{65}$.

Non era comunque l'adesione o meno alla politica doganale orientata in senso protezionista a tracciare le coordinate entro le quali la Milano economica si stava sempre più approssimando a quella di una città industriale. Del resto neppure associazioni di categoria e territoriali come quelle di cui si è appena detto e nelle quali si scomponeva ormai il nuovo assetto funzionale della Camera di Commercio, apparivano in grado di farlo in modo convincente. Bisognava mettersi in un'altra ottica, la stessa in cui aveva dichiarato di porsi il già ricordato Giuseppe Colombo, quando, da «tecnologo» di razza qual era, ne avrebbe scritto in occasione dell'Esposizione Nazionale di Milano, nel 1881, alla vigilia dell'apertura della ferrovia del Gottardo ${ }^{66}$.

In tale occasione infatti, egli non si era messo a celebrare l'avvento della grande industria, quasi che questa fosse ormai una scelta ineluttabile anche per Milano, così come lo era stata per Manchester, Sheffield, Rouen e Mulhouse ${ }^{67}$. Si sarebbe dovuto allora accettare che prendesse il sopravvento una struttura "pesante" della manifattura cittadina, fatta di vasti agglomerati di opifici e di addensamenti sempre maggiori di mano d'opera salariata, con il supporto di un rinnovato e potenziato assetto di iniziative del terziario, commerciali o bancarie che fossero ${ }^{68}$. Non era dunque quella, secondo Colombo, la Milano industriale da promuovere. Ce ne era invece un'altra incentrata su una "industria di dettaglio" ad ampio spettro, in grado di esprimersi attraverso un articolato complesso di specialità manifatturiere esercitate su piccola scala ${ }^{69}$. Queste, oltre ad assorbire una ingente massa di forza lavoro pur disseminata in unità produttive variamente configurate, venivano fatte acce-

65 Ivi, 26.

66 Per l'intervento di G. Colombo su Milano industriale apparso nel v. III di Mediolanum, la pubblicazione collettanea edita a Milano in tale occasione, se ne veda la versione pubblicata a cura di L. Cafagna, in Il Nord nella storia d'Italia. Antologia politica dell'Italia industriale, Bari, Laterza, 1962, 28-51.

67 C. Pavese, Dall'impresa privata all'azienda pubblica fra opportunità tecnologiche ed esplosione urbana: i primi settant'anni del trasporto pubblico milanese (18611931), in Id., A. Mantegazza (a cura di), L'ATM di Milano 1861-1972. Un secolo di trasporto urbano tra finalità pubbliche e vincoli di bilancio, Milano, F. Angeli, 1993, 36.

68 E. Borruso, Dal laboratorio artigiano alla piccola impresa urbana, cit., 80.

69 A. Colli, Piccole imprese e «piccole industrie» sino al 1945, in Storia d'Italia. L'industria, Annali 15, Torino, Einaudi, 1999. 
dere, come nel caso di Parigi portato ad esempio, alla variegata sfera del consumo tramite la loro stretta correlazione con le attività commerciali e creditizie di riferimento. Nè poteva essere diversamente, fintanto che non fosse stato sciolto il nodo energetico che attanagliava la città, a motivo della bassa quantità di forza motrice ad uso industriale assicurata dalle fonti idrauliche agibili entro il perimetro urbano ${ }^{70}$. Del resto sarebbe stato lo stesso Colombo a rendersi protagonista di una iniziativa imprenditoriale volta al superamento di tale vincolo, dando il proprio apporto fondamentale alla realizzazione nel 1898 della centrale idroelettrica di Paderno d'Adda, che faceva seguito a quella termoelettrica attivata nel 1883 a Milano in via Santa Redegonda, sempre grazie al suo intervento, questa volta ancor più pionieristico e decisivo ${ }^{71}$.

Se poi in questo modo egli aveva contribuito a porre le premesse per l'affermarsi della grande industria a Milano, ciò gli interessava relativamente. Aveva in effetti mostrato di continuare a credere che la sua città non fosse vocata allo sviluppo industriale ${ }^{72}$ se non nell'accezione tutta cattaneana che lo associava all'evolversi, dal suo interno, del proprio tessuto manifatturiero più tradizionale. Vi era però in tal caso indotto dal processo di un'intensa mercantilizzazione che andava investendo la struttura economica urbana nel suo complesso ${ }^{73}$. Il suo era del resto lo stesso percorso che Gino Luzzatto, tratteggiando le vicende dell'economia lombarda a 60 anni dall'Unità, avrebbe fatto proprio ${ }^{74} \mathrm{e}$ che più di recente Luciano Cafagna ci ha riproposto, reinterpretando la dinamica industriale milanese e lombarda come un processo "per ingrandimenti successivi", con ampia e costante convivenza del piccolo, del medio e del grande, in notevole autonomia reciproca ${ }^{75}$.

70 E. Borruso, Dal laboratorio artigiano alla piccola impresa urbana, cit., 80.

71 A. Moioli, Le vicende economiche dell'industria elettrica in Italia, in V. Cantoni, A. Silvestri (a cura di), Storia della tecnica elettrica, Milano, Cisalpino, 2005, 173.

72 A pag. 47 del suo scritto più sopra citato su Milano industriale, l'autore si domandava "Ma è necessario, è desiderabile che Milano diventi sede di grandi industrie?". La risposta era perentoria: "Noi non lo crediamo".

73 A. Moioli, Carlo Cattaneo e l'agricoltura lombarda, in Istituto Lombardo di Scienze e Lettere, Incontro di Studio n. 28, Cattaneo, Milano e la Lombardia, Milano, 2005, 133-134.

74 G. Luzzatto, L'evoluzione economica della Lombardia dal 1860 al 1922, cit., 464.

75 L. Cafagna, Bruno Caizzi e la storia dell'industria in Lombardia, in R. Ceschi, G. Vigo (a cura di), Tra Lombardia e Ticino. Studi in memoria di Bruno Caizzi, Bellinzona, Edizioni Casagrande, 1995, 20-21. 